\title{
Behavioral Development in the Absence of Neural Activity: Effects of Chronic Immobilization on Amphibian Embryos
}

\author{
Lanny J. Haverkamp and Ronald W. Oppenheim*,1 \\ Neurobiology Curriculum, University of North Carolina, Chapel Hill, North Carolina 27514, and *Department of \\ Anatomy, Wake Forest University, Bowman Gray School of Medicine, Winston-Salem, North Carolina 27103
}

Embryos of Xenopus laevis and Ambystoma mexicanum were continually immobilized from premotile stages of development to stages at which normally reared embryos were swimming well. Immobilization was achieved through exposure to solutions of chloretone, lidocaine, or alpha-bungarotoxin. At a number of stages after recovery from the drugs, spontaneous and stimulated behaviors were extensively quantified. Immobilization of Ambystoma embryos resulted in temporary defects in musculoskeletal development. In contrast, treated Xenopus embryos could not be distinguished from controls by simple visual observation within minutes to hours after removal from the drug solutions. Quantifications of behavior revealed, however, a transient period of 24-48 hr during which treated embryos exhibited consistently reduced measures of stimulated swimming, while showing an increase in frequency of spontaneous movements. Detailed behavioral testing could detect no permanent effects of chronic immobilization in either species after this initial period of recovery. The results are discussed in reference to the classic works of Harrison (1904), Carmichael (1926, 1927), and Matthews and Detwiler (1926).

Virtually all of the experimental evidence for the integral role of experience and function in neural development focuses on refinement within, or maintenance of, neuronal assemblies following a considerable amount of initial differentiation. The role of function and/or experience in the earliest stages of pathway development and synaptogenesis is largely unexplored. From the limited number of studies addressing this issue, there has evolved the widely held tenet that the nervous system develops in "forward reference" to, and without benefit from, function during the earliest stages of neurobehavioral development.

The principal in vivo evidence for this premise comes from the well-known studies of Harrison (1904), Carmichael (1926, 1927, 1928), and Matthews and Detwiler (1926). In all three sets of these classic works, amphibian embryos were immersed in solutions of an immobilizing drug prior to the onset of overt activity. When the animals were observed upon their removal from the drug solutions, their swimming behavior was judged to be normal. Thus, the conclusion was reached that "the mech-

\footnotetext{
Received May 16, 1985; revised Sept. 6, 1985; accepted Sept. 10, 1985.

We thank Kristin Edwards and Karen Wilson for technical assistance. The research reported here was supported by NIH Grants NS-16301 and NS-2042 to R. W. Oppenheim, with additional support derived from PHS Training Grant $\mathrm{MH} 14277$ (NIH) to the Neurobiology Program. Portions of this work have been published in abstract or book form (Haverkamp and Oppenheim. 1981; Oppenheim and Haverkamp, in press).

Correspondence should be addressed to L. J. Haverkamp, Dept. of Neurology, Baylor College of Medicine, 1 Baylor Plaza, Houston, TX 77030, his present address.

' Present address: Department of Anatomy, Bowman Gray School of Medicine, Wake Forest University, Winston-Salem, NC 27103.
}

Copyright (C) 1986 Society for Neuroscience $0270-6474 / 86 / 051332-06 \$ 02.00 / 0$ anism requisite for carrying out the complex muscular movements of locomotion and respiration develops normally without ever having functioned" (Harrison, 1904, p. 214).

Although this conclusion has been generally considercd valid, many of these early observations were of a cursory nature, while others were in seeming contradiction to the stated conclusions. Furthermore, only a single drug (chloretone), whose mode of action is largely unknown, was used to induce immobilization. These classic studies also provide only qualitative, observational data. The single replication of the paradigm that quantified the behavior of the embryos (Fromme, 1941) provides data leading to an opposite conclusion. Finally, it should be noted that the occurrence of apparently normal behavior following such treatment has led to the unwarranted inference that early neuronal development is also not regulated by functional activity.

The validity of the concept of "forward reference," if correct, would contribute an extremely important element to the understanding of neural and behavioral development. We have therefore replicated and extended the classic studies of Harrison and others. Xenopus laevis (South African clawed toad) embryos were immobilized by injection of alpha-bungarotoxin or by immersion in lidocaine or chloretone solutions. Observations and videotape records of several behavioral responses were made at a number of developmental stages after recovery or removal from the drug solutions. Quantitative measures of these behaviors, as well as qualitative observations, are discussed in reference to the earlier works. Neuroanatomical and physiological analyses of immobilized embryos will be presented in a companion paper (Haverkamp, 1986).

\section{Materials and Methods}

Fertilized eggs of Xenopus laevis were obtained by injecting adult pairs (NASCO, Ft. Atkinson, WI) with human chorionic gonadotropin. Within $9 \mathrm{hr}$ of fertilization, the eggs were placed in rearing solution (a $20 \%$ dilution of 3 parts Holtfreter's and 1 part Steinberg's media, pH 7.4; see Hamburger, 1960). All rearing, manipulation, and testing were done at $18-22^{\circ} \mathrm{C}$ under a 12:12 light-dark schedule. All stage notations refer to those of Nieuwkoop and Faber (1967).

The schedule of manipulation and testing of embryos is shown in Figure 1. At stage 17, embryos were randomly assigned to control or experimental groups and placed in aerated glass bowls of rearing solution, either with or without dissolved drug. Three drug solutions were used to induce immobilization: $0.6 \mathrm{~mm}$ lidocaine (Sigma), $1.7 \mathrm{~mm}$ chloretone (chlorobutanol; Sigma), and purified alpha-bungarotoxin ( $\alpha$ BTX; courtesy Dr. John Schmidt, SUNY-Albany). Approximately $2 \mu \mathrm{g}$ of $\alpha$-BTX was injected into stage 23 embryos in $0.5-1 \mu$ l of $0.09 \%$ saline plus methylene blue. Control animals for the $\alpha$-BTX series received injections of the saline-methylene blue solution.

At stage 35, chloretone- and lidocaine-immobilized embryos were removed from the drug solutions and placed in individual paraffincoated cups containing $50 \mathrm{ml}$ of rearing solution. Only those $\alpha$-BTXinjected embryos that showed first response to stimulation at stage 35 were retained for study and transferred similarly to individual containers 


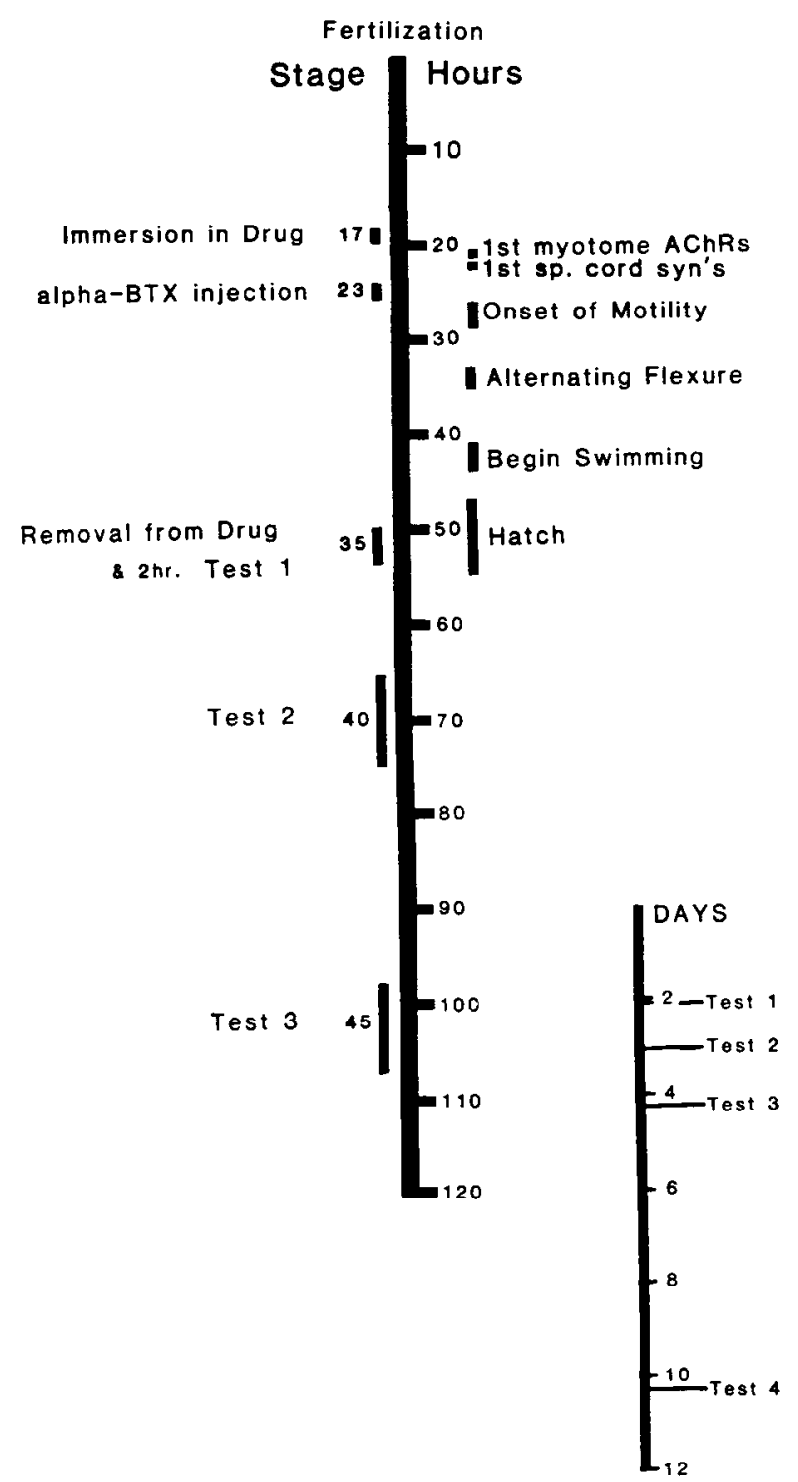

Figure 1. Timetable of Xenopus embryonic development. Nieuwkoop and Faber (1967) stages and schedule of drug application and behavioral testing on the left. Hours post-fertilization and some developmental landmarks on the right. Insert contains an expanded time scale to include the fourth test at stage 48 .

at this time. All comparisons between control and experimental animals were made between stage- and size-matched embryos from the same spawning. Behavioral observations were made both directly and by replay of videotape recordings.

Recordings of spontaneous movements were made of embryos maintained in individual cups, beginning at the moment of removal of the experimental animals from the drug solution (stage 35) for a continuous period of $48 \mathrm{hr}$ (approximately stage 45 ). These videotapes were replayed at $70 \times$ original speed, and the frequency and type of movements noted for each animal.

Videotape records of response to tactile stimulation were obtained by gently stroking each embryo with a pressure-calibrated hair mounted on a glass rod. Five such stimulations were given to each animal, 30 sec apart, at stages 35 ( $2 \mathrm{hr}$ after removal of experimental animals from the drug solution), 40, and 45 (see Fig. 1).

By stage 48 (approximately $7 \mathrm{~d}$ post-treatment), tactile stimulation was no longer feasible; therefore, stimulation was produced by lowfrequency vibration of the substrate (see Kimmel et al., 1980; Rock, 1980). The stimulus was imparted by the impact of a hand-activated solenoid on the hollow wooden platform on which the individual cups were resting. Ten minutes after moving the cups under the camera, the videotaping began with 3 min of spontaneous swimming followed by 25 stimulations at $5 \mathrm{sec}$ intervals, $1 \mathrm{~min}$ recovery, 120 stimulations at
1 sec intervals, and a final 3 min of spontaneous swimming.

One additional group of five embryos was maintained in normal rearing solution until stage 35 , when they were transferred to lidocaine solution. At stage 40 , these animals were replaced in normal rearing solution, tested $2 \mathrm{hr}$ later, and retested at stages 45 and 48 . Equal numbers of embryos from the same spawning, raised either in normal rearing solution or in lidocaine from stages 17 to 35 , were tested at the same times for these comparisons.

A single experiment was performed on axolotl embryos (Ambystoma mexicanum; courtesy of G. M. Malacinski, Axolotl Colony, Indiana University). At stages 23-25 (Harrison, 1969), a premotile stage of development, 45 embryos were randomly selected for rearing in either normal rearing solution or $1.9 \mathrm{~mm}$ chloretone. Approximately one-half of the animals of each group were decapsulated at this time while the rest were left undisturbed until the time of removal from the drug. At stage $38 / 39$ and stage $39 / 40$ ( 7 and $9 \mathrm{~d}$ after immersion), separate groups of embryos were transferred from the chloretone solution to normal rearing solution. Observations and testing of both of these groups, as well as of control embryos, were performed $2 \mathrm{hr}$ after removal from the drug and again $1,3,5,8$, and $10 \mathrm{~d}$ later.

Behavioral responses of both species were quantified by viewing a computer-digitizing tablet through the mounted components of a camera lucida, thus superimposing the image of a video monitor on the surface of the tablet. After calibrating the tablet to the known cup size $(6 \mathrm{~cm}$ outside diameter), the path each animal swam was traced on the tablet as the videotape record of the embryo's responses was replayed at reduced speed. The $x-y$ coordinates of the position of the embryo were relayed to the computer at $0.083 \mathrm{sec}$ intervals. From these data were derived the path traversed in response to stimulation, as well as the duration, distance, and mean speed of the entire response and portions thereof. The mean values of the above measures were calculated for each animal and for each experimental group, and analyzed by a one-tailed Mann-Whitney $U$ test. All statements of statistical significance refer to a probability value of less than 0.05 .

\section{Results}

Qualitative observations of the Xenopus embryos' recovery from immobilization were essentially in agreement with data published in the earlier works. Initial responses to stimulation were seen as early as $2-10 \mathrm{~min}$ after removal from the chloretone solutions, or as late as $60 \mathrm{~min}$ after removal from lidocaine. By $2 \mathrm{hr}$ after removal from chloretone, direct visual observation of experimental animals could not distinguish them from controls. Similar degrees of recovery were not achieved by lidocaine-reared embryos for approximately $16 \mathrm{hr}$, and by $\alpha$-BTXinjected embryos for 3-6 hr after first response to stimulation.

Graphs comparing the speed, distance, and duration of swiming of experimental and control embryos are shown in Figure 2. Deficits in distance and speed of swimming were detectable during testing $2 \mathrm{hr}$ after removal from either chloretone or lidocaine solutions. For instance, chloretone-reared animals swam with an average speed of $21.9 \mathrm{~mm} / \mathrm{sec}$ (vs $41.0 \mathrm{~mm} / \mathrm{sec}$ for controls) for a mean distance per stimulated bout of $19.9 \mathrm{~mm}$ (vs $41.2 \mathrm{~mm}$ ). By stage 40 , the chloretone-reared and $\alpha$-BTXinjected animals showed no differences from control embryos, although lidocaine-reared animals remained less responsive. At stage 45 (approximately $48 \mathrm{hr}$ after release from the drugs), all experimental animals responded normally. Other measures, i.e., speed of swimming during the first 0.08 and the first $0.16 \mathrm{sec}$ after stimulation, and of maximum speed achieved in each swimming bout, were also transiently reduced at those stages of testing during which the graphed measures were depressed.

At stage 48 (about $7 \mathrm{~d}$ after release), all experimental animals remained equivalent to control animals in their responses to all aspects of the vibratory stimulation series (Fig. 3).

The frequency of spontaneous movement of animals reared in either chloretone or lidocaine solutions was significantly greater than that of control animals during the initial portions of the $48 \mathrm{hr}$ observation periods (Fig. 4). There were no differences, however, in the degree to which different types of activity were spontaneously performed (i.e., single twitches or coils vs swimming bouts; see Coghill, 1929). 


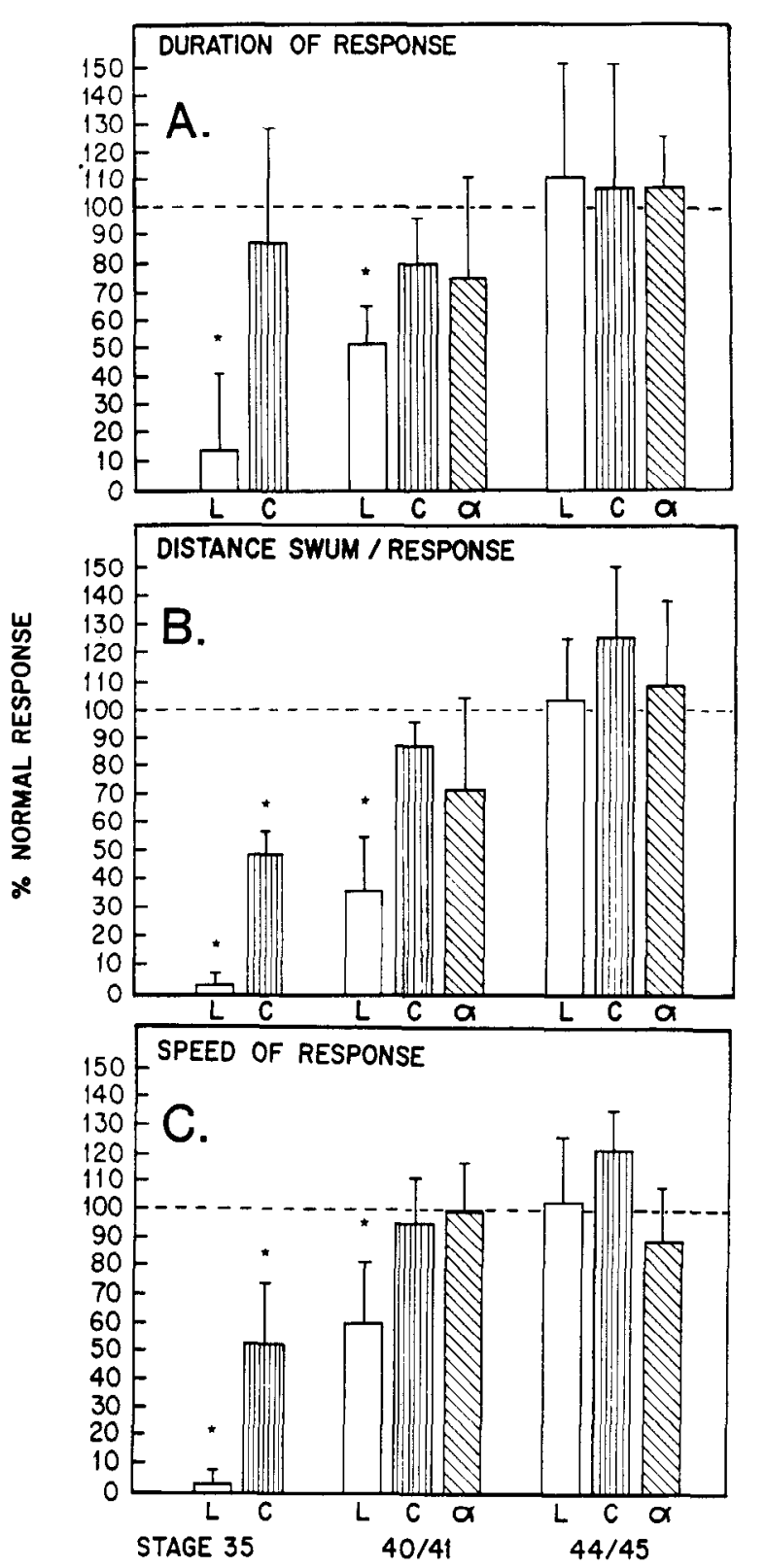

Figure 2. Quantitative measures of response to tactile stimulation by Xenopus embryos, $2 \mathrm{hr}$ after removal from the drug solution (stage 35 ), approximately $24 \mathrm{hr}$ later (stage $40 / 41$ ), and approximately $48 \mathrm{hr}$ after removal from the drug solution (stage 44/45). $C=$ chloretone-immobilized stages 17-35 $(n=15) ; L=$ lidocaine-immobilized stages 17-35 $(n=12) ; \alpha=$ immobilized by injection of $\alpha$-BTX at stage $23(n=8)$. Asterisk, Values that differ significantly from those of normally reared embryos. $A$, Mean duration of response to tactile stimulation. $B$, Mean distance swum in response to stimulation. $C$, Mean speed of stimulated response.

Immobilization of embryos after they had begun effective swimming resulted in transient effects on behavior similar to those seen in embryos immobilized during earlier stages. Two hours after removal from lidocaine, embryos immobilized from stage 35 to 40 swam more slowly and for shorter distances than normal embryos, but were equivalent in these measures to stage 17-35-immobilized animals (which had been in normal rearing solution for approximately $24 \mathrm{hr}$ ). At stages 45 and 48 , the lateimmobilized animals were indistinguishable from both the normally reared and the stage 17-35-immobilized embryos.

For a period of several days after removal of axolotl embryos from the drug solution, those embryos not released from their

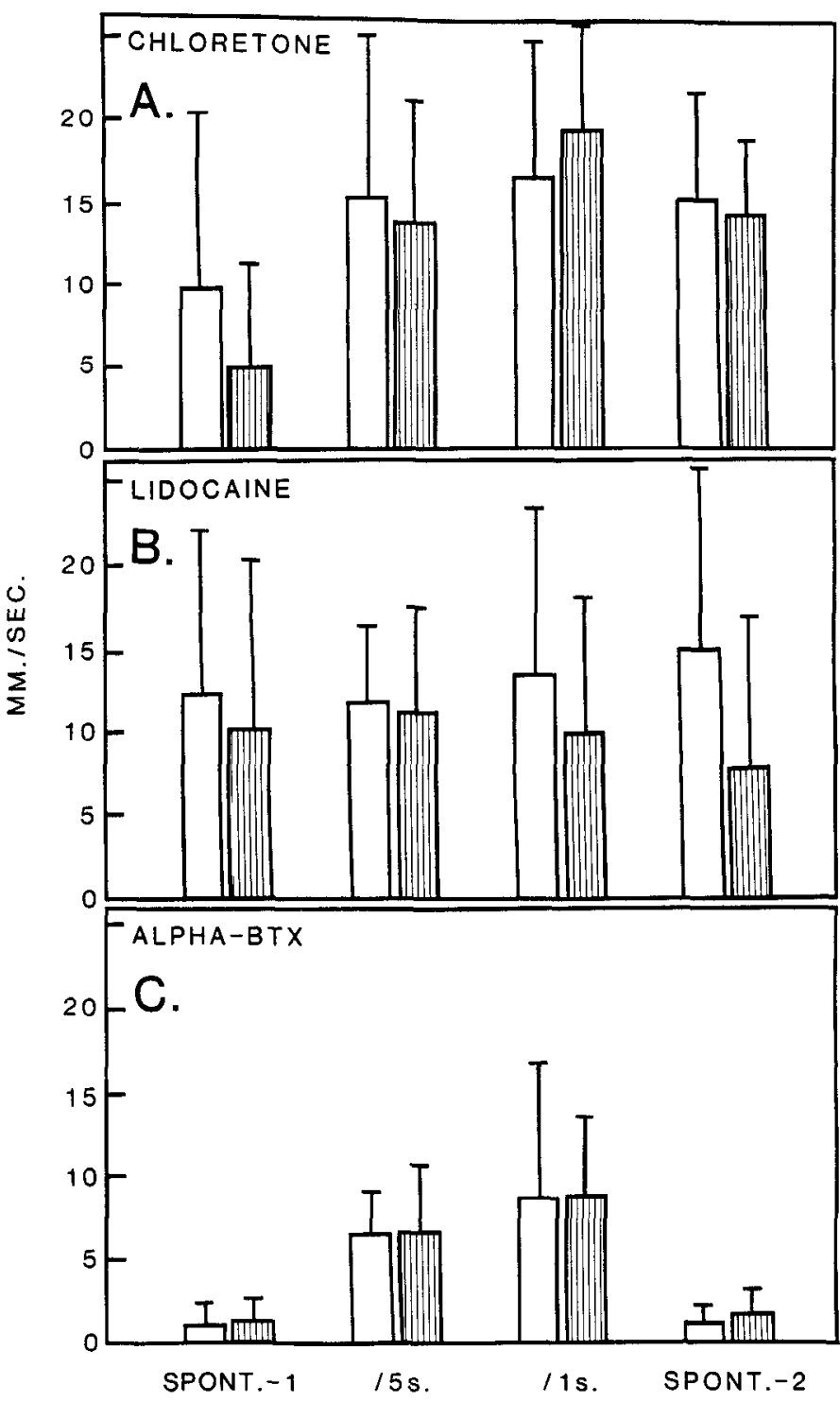

Figure 3. Swimming speed of stage 48 Xenopus tadpoles $(7 \mathrm{~d}$ after removal from drug solution) during vibratory stimulation testing series. Spont.-1, Initial 3 min spontaneous swimming period; $/ 5 \mathrm{~s}$., $125 \mathrm{sec}$ period, with stimuli applied at $5 \mathrm{sec}$ intervals; $/ 1 \mathrm{~s}$., $125 \mathrm{sec}$ period, with stimuli applied at 1 sec intervals; Spont. -2 , final 3 min spontaneous swimming period. Open bars, Control (normally reared) embryos; shaded bars, immobilized embryos. None of the differences between control and immobilized embryos are statistically significant. $A$, Chloretoneimmobilized $(n=15)$. $B$, Lidocaine-immobilized $(n=12) . C, \alpha$-BTX immobilized $(n=8)$.

egg capsules at the onset of immobilization retained the body curvature imposed upon them by the restraining membranes; those that had been decapsulated before chloretone immersion did not exhibit this curvature. When the videotapes of these axolotls were replayed at reduced speed, it became apparent that both groups of previously immobilized embryos were restricted and less fluid in their movements than were control animals. This was most apparent in the curved animals, for which the postural asymmetry and trunk rigidity resulted in a tendency to swim in a circular pattern. Over 4-10 d after their transfer to normal rearing solution the curvature of these animals gradually subsided, and all of the immobilized axolotls developed more fluidity in their movements. Measurements of speed and distance of swimming of the immobilized animals were consistently less than those of controls during the period of structural deformity. Tests at 8 and $10 \mathrm{~d}$ after removal from chloretone, 


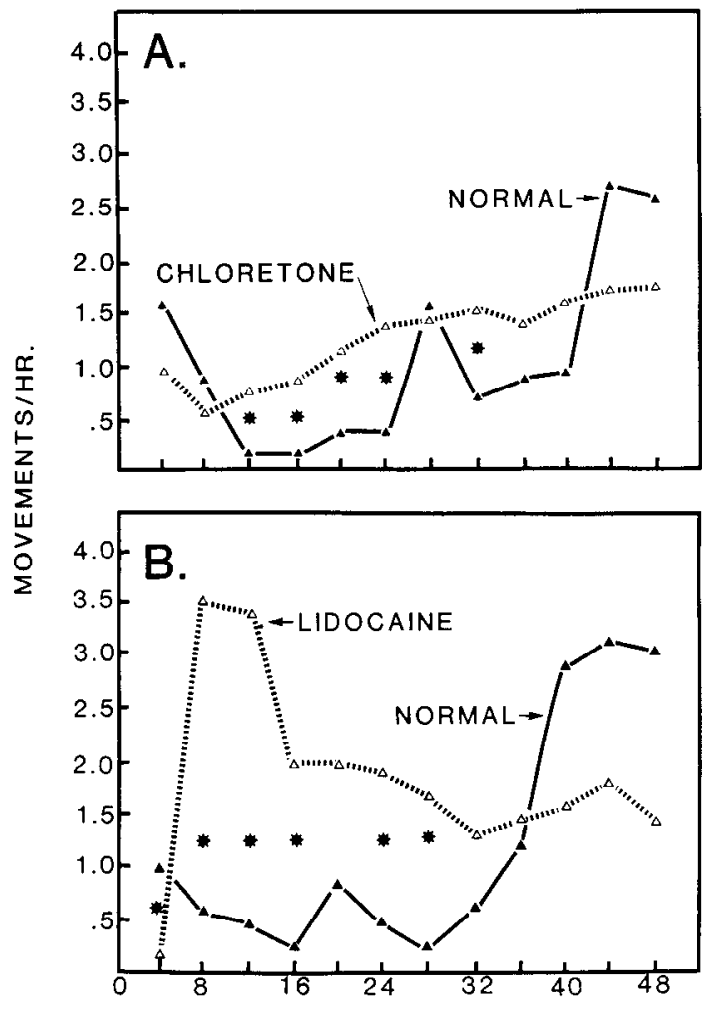

HOURS AFTER DRUG RELEASE (ST.35)

Figure 4. Spontaneous activity of normally reared and immobilized (stages 17-35) Xenopus embryos. The $48 \mathrm{hr}$ observation period began at the time of removal of the immobilized embryos from the drug solution (stage 35). $n=10$ for each group. Asterisk, Points at which the two values differ significantly. $A$, Chloretone-immobilized. $B$, Lidocaineimmobilized.

when structural defects had abated, revealed no significant differences from control animals.

\section{Discussion}

At stage 17, synapses have not yet formed within the Xenopus spinal cord, and the first neuromuscular junctions and overt movements do not appear for another 5-7 hr (Blackshaw and Warner, 1976; Hayes and Roberts, 1973). Yet, when all neural activity is pharmacologically blocked from this stage of development until the time of hatching, embryos behave in an apparently normal manner within minutes to hours after their removal from the blocking agents. Within $24-48 \mathrm{hr}$ after removal of the drug block, even detailed quantitative measures of behavior can no longer distinguish treated from normally reared animals. Thus, we conclude that the standard interpretation of the early classic studies (Carmichael, 1926, 1927; Harrison, 1904; Matthews and Detwiler, 1926) is valid. Preventing neural impulse activity during the earliest stages of motor system development does not interfere with the later function of that system.

There have been a number of conceptually related studies, performed primarily in vitro, since Harrison's (1904) pioneering work. Although developmental abnormalities resulting from inhibition of neural activity have been demonstrated in a number of systems (Bergey et al., 1981; Christian et al., 1980; Janka and Jones, 1982), the ability of neural tissue to develop normally, seen in other studies (Bird, 1980; Crain, 1980; Crain ct al., 1968; Harris, 1980; Model et al., 1971; Romijn et al., 1981), is apparently sufficient for the exhibition of normal behavioral output as evidenced in the present work.

The early works of Harrison, Carmichael, and Matthews and
Detwiler have remained, however, the best in vivo evidence for the initial lack of involvement of functional activity in neurobehavioral development. The studies reported here have extended their paradigm and addressed some of the issues raised by a critical reading of those reports.

The major shortcoming to interpretation of these early studies is their lack of quantification. Quantitative measurements of the behavior of Rana embryos, reared under the same conditions, led Fromme (1941) to an opposite conclusion, i.e., that blockade of neural activity early in development does have an effect upon later behavior. Unfortunately, Fromme's (1941) study did not include any observations made beyond $1 \mathrm{hr}$ after removal from the drug solution. In the present study this early effect of immobilization on quantitative aspects of swimming behavior was reproduced (at $2 \mathrm{hr}$ after removal from the drug), but the effect was shown to be a transient one. Chloretone-immobilized animals were quantitatively indistinguishable from normal animals within $24 \mathrm{hr}$ after release. Because quantitative measures were not made between 2 and $24 \mathrm{hr}$, it is not possible to conclude when it was during this $22 \mathrm{hr}$ period that the deficits subsided.

A second difficulty in accepting the common interpretations of the early works was the unknown mode of action of the immobilizing agent, chloretone. While the mode of action of chloretone is still uncertain, recent studies indicate that its immobilizing effects are due, at least in part, to effects within the central nervous system. ${ }^{2}$ Lidocaine has been clearly shown to be a conduction blocker in both central and peripheral fibers (e.g., Courtney et al., 1978; Crain et al., 1968). $\alpha$-BTX is a competitive inhibitor of nicotinic cholinergic receptors, acting primarily at the neuromuscular junction (e.g., Lee et al., 1977), though L. Landmesser (personal communication) has suggestive evidence that this drug may also act centrally in the chick embryo. Although the time course of behavioral recovery was more prolonged after lidocaine immersion than after exposure to either chloretone or $\alpha$-BTX, full quantitative recovery was nevertheless achieved after treatment with any of the drugs.

A final problem with these early studies was the sufficiency of the reported data as justification for the conclusions drawn. Although the recorded observations of Harrison (1904) and Carmichael $(1926,1927)$ were neither detailed nor extensive, their stated conclusions are in full agreement with those reached in this study. The work of Matthews and Detwiler (1926) was certainly detailed, yet the time course for recovery from immobilization (5-14 d, depending on drug concentration and duration of exposure) was extremely prolonged. Since the experimental animal used by Matthews and Detwiler was the salamander, Ambystoma punctatum, an insight into this extended drug effect may be seen in the data obtained here for the axolotl, A. mexicanum. Matthews and Detwiler removed all constraining egg capsules from experimental animals prior to their immersion in the drug solution. Thus, one can assume that after removal from the drug the animals' resting posture appeared normal (i.e., straight) and that the severe and long-lasting curvature of immobilized animals that remained encapsulated was not observed. The truncal rigidity of immobilized Ambystoma reported in the present study is not readily apparent by simple observation of animals that do not possess a postural asymmetry. It seems likely, therefore, that the results of Matthews

\footnotetext{
${ }^{2}$ Edwards and Larrabee (1955) have shown that chloretone produces a decrement in impulse propagation along isolated preganglionic nerves of the rat superio cervical ganglion. However, W. A. Harris (personal communication) found chloretone to be without effect on propagation of the compound action potential in desheathed adult Xenopus sciatic nerve. In the companion paper to this work (Haverkamp, 1986), chloretone is shown to completely inhibit the ventral root activity that results from mechanical stimulation of Xenopus embryos. Thus, while it is still unclear by what means chloretone acts in amphibian embryos, its site of action most likely includes portions of the CNS, and does not produce immobility through simple block of the neuromuscular junction.
} 
and Detwiler (1926) may bc duc in part to an unnoticed abnormal development of the musculoskeletal system of their experimental animals. Long-term, chronic immobilization is known to result in similar defects in chick embryos (Drachman and Sokoloff, 1966; Oppenheim et al., 1978).

The initial period of behavioral retardation seen in Xenopus embryos after long-term immobilization may have been due to similar, though unobserved, effects, which resulted in a mechanical restriction of movement. ${ }^{3}$ Developmental arrest of certain other nonneural systems (e.g., Armstrong et al., 1983) may also have contributed to the behavioral deficits seen during this period.

It seems most likely, however, that these transient decreases in stimulated behavior were due to the effects of residual drug. The fact that embryos that were immobilized after they had already begun to swim (during stages 35-40) showed a similar transient period of behavioral deficit indicates that such effects are not specific to drug exposure during the earliest stages of development. The initially elevated levels of spontaneous movement by previously immobilized embryos might be ascribed to the increases in neural activity seen during recovery from a number of anesthetizing agents (e.g., Roth, 1980). Such a relation would support the notion that removal of the drugs or their metabolites is a prolonged process. The frequency-dependent nature of lidocaine conduction block at certain drug concentrations (Courtney, 1975; Courtney et al., 1978; Trubatch, 1972) may provide insight into the observed increase in spontaneous activity during the time in which measures of stimulated behaviors are depressed.

Finally, there is the possibility that although behavior is unaffected, early immobilization does, indeed, result in abnormal neural development. For example, embryonic paralysis of the chick results in a near-doubling of the number of motor neurons innervating the limbs; however, many aspects of chick motor performance appear unaffected after such treatment (Oppenheim, 1984; Oppenheim et al., 1978; Pittman and Oppenheim, 1979). It might also be argued that the transience of Xenopus swimming defects following immobilization is the result of a rapid compensation for drug-induced neural defects. There are, however, no effects at any time point on the output of the neuronal assemblies that produce swimming behavior. Even during the period of behavioral depression following immobilization, the proximate neural basis of swimming, i.e., the electrical activity of the ventral roots, is comparable to that of normal animals (Haverkamp, 1983, 1986).

Spontaneous embryonic motility has been demonstrated in all forms in which it has been investigated (Coghill, 1940; Corner, 1978; Corner et al., 1979; Hamburger, 1963, 1973; Oppenheim, 1974, 1982), and its contribution to the normal development of a number of nonneuronal systems has been established (Drachman and Sokoloff, 1966; Herring and Lakars, 1981; Oppenheim et al., 1978; Urch and Hedrick, 1981). Amphibian and fish embryos are unique in that the earliest forms of this activity can be seen as direct precursors to the animals' initial adaptive behavioral performances, such as swimming (e.g., see Coghill, 1929; Tracy, 1926). This temporal progression of behavioral pattern makes it possible to assess directly the contributions of antecedent embryonic movements to the effective performance of later behaviors. Whilc the experiments presented above do not demonstrate the complete lack of behavioral consequences of blockade of embryonic activity, they do show that such effects as can be identified are transient and,

\footnotetext{
${ }^{3}$ Preliminary experiments were performed to compare degrees of body flexion during swimming in Xenopus. Although the techniques used to approach that study proved inadequate to provide acceptably accurate measures, the data derived did not indicate any differences between control and experimental groups. These preliminary findings imply that the musculoskeletal rigidity seen in other species was not a factor in the swimming of previously immobilized Xenopus embryos.
}

possibly, artifactual, and that detailed quantifications reveal no permanent effects on behavioral development. Thus, at least for amphibians, it can be concluded that the activation of those movements that normally precede the occurrence of a fundamental behavioral pattern such as swimming are not necessary for the normal development and performance of that behavior. In a companion paper, it is shown that certain neuroanatomical and neurophysiological correlates of swimming are also unaffected by such treatment (Haverkamp, 1986).

\section{References}

Armstrong, D. L., L. Turin, and A. E. Warner (1983) Muscle activity and the loss of electrical coupling between striated muscle cells in Xenopus embryos. J. Neurosci. 3: 1414-1421.

Bergey, G. K., S. C. Fitzgerald, B. K. Schrier, and P. G. Nelson (1981) Neuronal maturation in mammalian cell culture is dependent on spontaneous electrical activity. Brain Res. 207: 49-58.

Bird, M. M. (1980) The morphology of synaptic profiles in explants of foetal and neonatal mouse cerebral cortex maintained in a magnesium-enriched environment. Cell Tissue Res. 206: 115-122.

Blackshaw, S., and A. E. Warner (1976) Onset of acetylcholine sensitivity and endplate activity in developing myotome muscles of Xenopus. Nature 262: 217-218.

Carmichael, L. (1926) The development of behavior in vertebrates experimentally removed from the influence of external stimulation. Psychol. Rev. 33: 51-58.

Carmichael, L. (1927) A further study of the development of behavior in vertebrates experimentally removed from the influence of external stimulation. Psychol. Rev. 34: 34-47.

Carmichael, L. (1928) A further study of the development of behavior. Psychol. Rev. 35: 253-260.

Christian, C. N., G. K. Bergey, M. P. Daniels, and P. G. Nelson (1980) Cell interactions in nerve and muscle cell cultures. J. Exp. Biol. 89: 85-101.

Coghill, G. E. (1929) Anatomy and the Problem of Behavior. Cambridge U. P., London.

Coghill, G. E. (1940) Early embryonic somatic movements in birds and in mammals other than man. Monogr. Soc. Res. Child Dev. 5: $1-48$.

Corner, M. (1978) Spontaneous motor rhythms in early life-Phenomenological and neurophysiological aspects. Prog. Brain Res. 48: 349-364.

Corner, M. A., H. L. Bour, and M. Mirmiran (1979) Development of spontaneous motility and its physiological interpretation in the rat, chick, and frog. In Neural Growth and Differentiation, E. Meisami and M. A. B. Brazier, eds., pp. 253-267, Raven, New York.

Courtney, K. R. (1975) Mechanism of frequency-dependent inhibition of sodium currents in frog myelinated nerves by the lidocaine derivative GEA 968. J. Pharmacol. Exp. Ther. 195: 225-236.

Courtney, K. R., J. J. Kendig, and E. N. Cohen (1978) Frequencydependent conduction block: The role of nerve impuise pattern in local anesthetic potency. Anesthesiology 48: 111-117.

Crain, S. M. (1980) Development of specific scnsory-croked synaptic networks in organized CNS tissue cultures. In Tissue Culture in Neurobiology, E. Giacobini, A. Vernadakis, and A. Shahar, eds., pp. 169185, Raven, New York.

Crain, S. M., M. B. Bornstein, and E. R. Peterson (1968) Maturation of cultured embryonic CNS tissues during chronic exposure to agents which prevent bioelectric activity. Brain Res. 8: 363-372.

Drachman, D. B., and L. Sokoloff (1966) The role of movement in embryonic joint development. Dev. Biol. 14: 401-420.

Edwards, C., and M. G. Larrabee (1955) Effects of anaesthetics on metabolism and on transmission in sympathetic ganglia of rats: Measurement of glucose in microgram quantities using glucose oxidase. J. Physiol. (Lond.) 130: 456-466.

Fromme, A. (1941) An experimental study of the factors of maturation and practice in the behavioral development of the embryo of the frog Rana pipiens. Genet. Psychol. Monogr. 24: 219-256.

Hamburger, V. (1960) A Manual of Experimental Embryology. U. Chicago P., Chicago, IL.

Hamburger, V. (1963) Some aspects of the embryology of behavior. Q. Rev. Biol. 38: 342-365.

Hamburger, V. (1973) Anatomical and physiological basis of embryonic motility in birds and mammals. In Studies on the Development 
of Behavior and the Nervous System, Vol. I: Behavioral Embryology, G. Gottlieb, ed., pp. 51-76, Academic, New York.

Harris, W. A. (1980) The effects of eliminating impulse activity on the development of retinotectal projection in salamanders. J. Comp. Neurol. 194: 303-317.

Harrison, R. G. (1904) An experimental study of the relation of the nervous system to the developing musculature in the embryo of the frog. Am. J. Anat. 3: 197-220.

Harrison, R. G. (1969) Harrison stages and description of the normal development of the spotted salamander, Amblystoma punctatum (Linn.). In Organization and Development of the Embryo, S. Wilens, ed., pp. 44-66, Yale U. P., New Haven, CT.

Haverkamp, L. J. (1983) Neurobehavioral development with blockade of neural function in embryos of Xenopus laevis. Ph.D. dissertation, University of North Carolina, Chapel Hill, NC.

Haverkamp, L. J. (1986) Anatomical and physiological development of the Xenopus embryonic motor system in the absence of neural activity. J. Neurosci. $6 ; 1338-1348$.

Haverkamp, L. J., and R. W. Oppenheim (1981) Effects of neural blockade on behavioral development in Xenopus embryos. Soc. Neurosci. Abstr. 7: 181.

Hayes, B. P., and A. Roberts (1973) Synaptic junction development in the spinal cord of an amphibian embryo: An electron microscope study. Z. Zellforsch. 137: 251-269.

Herring, S. W., and T. C. Lakars (1981) Craniofacial development in the absence of muscle contraction. J. Craniofacial Genet. Devel. Biol. 1: $341-357$

Janka, Z., and D. G. Jones (1982) Junctions in rat neocortical explants cultured in TTX-, GABA-, and $\mathrm{Mg}^{++}$-environments. Brain Res. Bull. 8: 273-278.

Kimmel, C. B., R. C. Eaton, and S. L. Powell (1980) Decreased faststart performance of zebrafish larvae lacking Mauthner neurons. J. Comp. Physiol. 140: 343-450.

Lee, C., D. Chen, and R. L. Katz (1977) Characteristics of nondepolarizing neuromuscular block: (I) Post-junctional block by alphabungarotoxin. Can. Anaesth. Soc. J. 24: 212-219.

Matthews, S. A., and S. R. Detwiler (1926) The reaction of Amblystoma embryos following prolonged treatment with chloretone. J. Exp. Zool. 45: 279-292.

Model, P. G., M. B. Bornstein, S. M. Crain, and G. D. Pappas (1971) An electron microscopic study of the development of synapses in cultured fetal mouse cerebrum continuously exposed to xylocaine. J. Cell Biol. 49: 362-371.
Nieuwkoop, P. D., and J. Faber (1967) Normal Table of Xenopus laevis, 2nd ed., North Holland, Amsterdam.

Oppenheim, R. W. (1974) The ontogeny of behavior in the chick embryo. In Advances in the Study of Behavior. Vol. 5, D. S. Lehrman, R. A. Hinde, E. Shaw, and J. Rosenblatt, eds., pp. 133-172, Academic, New York.

Oppenheim, R. W. (1982) The neuroembryological study of behavior: Progress, problems, perspectives. Curr. Topics Dev. Biol. 17: 257309.

Oppenheim, R. W. (1984) Ccll death of motoneurons in the chick embryo spinal cord. VIII. Motoneurons prevented from dying in the embryo persist after hatching. Dev. Biol. 101: 35-39.

Oppenheim, R. W., and L. J Haverkamp (in press) Early development of behavior and the nervous system: An embryological perspective. In Developmental Processes in Psychobiology and Neurobiology, E. M. Blass, ed., Plenum, New York.

Oppenheim, R. W., R. Pittman, M. Gray, and J. L. Maderdrut (1978) Embryonic behavior, hatching and neuromuscular development in the chick following a transient reduction of spontaneous motility and sensory input by neuromuscular blocking agents. J. Comp. Neurol. 179: 619-640.

Pittman, R., and R. W. Oppenheim (1979) Cell death of motoneurons in the chick embryo spinal cord. IV. Evidence that a functional neuromuscular intcraction is involved in the regulation of naturally occurring cell death and the stabilization of synapses. J. Comp. Neurol. 187: 425-446.

Rock, M. K. (1980) Functional properties of Mauthner cell in the tadpole Rana catesbeiana. J. Neurophysiol. 44: 135-150.

Romijn, H. J., M. T. Mud, A. M. M. C. Habets, and P. S. Wolters (1981) A quantitative electron microscopic study on synapse formation in dissociated fetal rat cerebral cortex in vitro. Dev. Brain Res. 1: 591-605.

Roth, S. H. (1980) Differential effects of anesthetics on neuronal activity. Prog. Anesthesiol. 2: 119-128.

Tracy, H. C. (1926) The development of motility and behavior reactions in the toadfish (Opsanus tau). J. Comp. Neurol. 40: 253-369.

Trubatch, J. (1972) Transmission of high-frequency trains of impulses in normal and procainized frog nerve. Am. J. Physiol. 223: 637-643.

Urch, U. A., and J. L. Hedrick (1981) The hatching enzyme from Xenopus laevis: Limited proteolysis of the fertilization envelope. $\mathrm{J}$. Supramol. Struct. Cell. Biochem. 15: 111-117. 